\title{
Detecting the molecular scars of evolution in the Mycobacterium tuberculosis complex by analyzing interrupted coding sequences
} Caroline Deshayes ${ }^{1,2}$, Emmanuel Perrodou ${ }^{3}$, Daniel Euphrasie ${ }^{1}$, Eric Frapy ${ }^{1,2}$, Olivier Poch ${ }^{3}$, Pablo Bifani ${ }^{4}$, Odile Lecompte ${ }^{3}$ and Jean-Marc Reyrat*1,2

\author{
Address: ${ }^{1}$ Université Paris Descartes, Faculté de Médecine René Descartes, Paris Cedex 15, F-75730, France, ${ }^{2}$ Inserm, U570, Unité de Pathogénie \\ des Infections Systémiques, Paris Cedex 15, F-75730, France, ${ }^{3}$ Laboratoire de Biologie et Génomique Structurales, IGBMC CNRS/INSERM/ULP, \\ BP 163, 67404 Illkirch Cedex, France and ${ }^{4}$ Institut Pasteur de Bruxelles, Laboratoire Tuberculose et Mycobactéries, Brussels, Belgium \\ Email: Caroline Deshayes - deshayes@necker.fr; Emmanuel Perrodou - perrodou@titus.u-strasbg.fr; Daniel Euphrasie - euphrasi@necker.fr; \\ Eric Frapy - frapy@necker.fr; Olivier Poch - poch@titus.u-strasbg.fr; Pablo Bifani - pbifani@pasteur.be; \\ Odile Lecompte - odile.lecompte@titus.u-strasbg.fr; Jean-Marc Reyrat* - jmreyrat@necker.fr \\ * Corresponding author
}

Published: 6 March 2008

BMC Evolutionary Biology 2008, 8:78 doi:10.1186/147/-2148-8-78

This article is available from: http://www.biomedcentral.com/I47|-2/48/8/78

(C) 2008 Deshayes et al; licensee BioMed Central Ltd.

This is an Open Access article distributed under the terms of the Creative Commons Attribution License (http://creativecommons.org/licenses/by/2.0), which permits unrestricted use, distribution, and reproduction in any medium, provided the original work is properly cited.
Received: 23 August 2007

Accepted: 6 March 2008

\begin{abstract}
Background: Computer-assisted analyses have shown that all bacterial genomes contain a small percentage of open reading frames with a frameshift or in-frame stop codon We report here a comparative analysis of these interrupted coding sequences (ICDSs) in six isolates of M. tuberculosis, two of $M$. bovis and one of $M$. africanum and question their phenotypic impact and evolutionary significance.

Results: ICDSs were classified as "common to all strains" or "strain-specific". Common ICDSs are believed to result from mutations acquired before the divergence of the species, whereas strainspecific ICDSs were acquired after this divergence. Comparative analyses of these ICDSs therefore define the molecular signature of a particular strain, phylogenetic lineage or species, which may be useful for inferring phenotypic traits such as virulence and molecular relationships. For instance, in silico analysis of the W-Beijing lineage of $M$. tuberculosis, an emergent family involved in several outbreaks, is readily distinguishable from other phyla by its smaller number of common ICDSs, including at least one known to be associated with virulence. Our observation was confirmed through the sequencing analysis of ICDSs in a panel of $2 \mathrm{I}$ clinical M. tuberculosis strains. This analysis further illustrates the divergence of the W-Beijing lineage from other phyla in terms of the number of full-length ORFs not containing a frameshift. We further show that ICDS formation is not associated with the presence of a mutated promoter, and suggest that promoter extinction is not the main cause of pseudogene formation.

Conclusion: The correlation between ICDSs, function and phenotypes could have important evolutionary implications. This study provides population geneticists with a list of targets, which could undergo selective pressure and thus alters relationships between the various lineages of $M$. tuberculosis strains and their host. This approach could be applied to any closely related bacterial strains or species for which several genome sequences are available.
\end{abstract}




\section{Background}

Recent in silico surveys showed that most bacterial genomes contain interrupted coding sequences (ICDSs) [1-3]. These ICDSs generally result from the insertion or deletion of nucleotides, affecting the frame read and splitting the original coding sequence into two or more smaller open reading frames. These mutations may also result in a shift in reading frame, thereby altering the carboxy-terminus of the protein. ICDSs may be present in genes with known or unknown functions, or in hypothetical open reading frames [4]. Reported prokaryotic genomes have a mean of 74 ICDSs per genome, corresponding to 1 to $5 \%$ of the genes present, irrespective of genome size or GC content [2,3]. One of the few exceptions is the genome of $M$. leprae, which contains about $30 \%$ ICDSs, frequently described as pseudogenes $[2,5]$. The accumulation of mutations in this species is thought to be due to the loss of the proofreading activity of the DnaQ subunit of DNA polymerase III [6]. A similar sort of reductive evolution is also observed in the case of $M$. ulcerans [7] or for species of the genus Rickettsiales [8]. ICDSs may correspond to authentic mutations, generally resulting in a loss of function, but may in some cases reflect sequencing errors. These sequencing errors are misleading when conducting genomic analysis, but have been shown to account for only some of the detected ICDSs [4,9-12]. Most ICDSs correspond to authentic mutations and can therefore be compared between strains, making it possible to explore conserved and unique mutation events.

The availability of complete genomes sequences for genetically related organisms has facilitated comparative analyses of ICDSs. This simple concept, which has not been reported before, enables to investigate evolutionary relationship between isolates or species. In this study, we took the finished genome of two mycobacterial species as a model: M. tuberculosis, which causes tuberculosis in humans, and M. bovis, which principally causes tuberculosis in ruminants. We also studied six phylogenetically distinct isolates of $M$. tuberculosis - H37Rv, CDC1551, Haarlem, F11, C [13], and 210 (a representative of the WBeijing family) and M. africanum, a species of the M. tuberculosis complex for which the genome sequence is still at the assembly step. These isolates are different from each other as they belong to distinct evolutionary branches of the M. tuberculosis species, sensu stricto (s.s), yet more closely related to each other than to the more distantly related members of the $M$. tuberculosis complex (M. africanum, M. bovis, M. microti and M. pinnipedii) [14]. The WBeijing family is a clonal group of highly successful $M$. tuberculosis strains associated with multiple outbreaks [15]. This family is one of the oldest lineages to diverge as determined by single nucleotide polymorphism (SNP) and region of deletion analysis [14]. In contrast, H37Rv, the first M. tuberculosis strain to be completely sequenced is believed to be one of the most recent (youngest) lineages of M. tuberculosis [14,16]. Strain CDC1551 belongs to a lineage that branched between the W-Beijing and the $\mathrm{H} 37 \mathrm{Rv}$ isolates. Overall these three isolates represent 3 different genetic groups of the species [14-17]. These isolates have been studied in detail and display differences in genotype $[14,18]$, phenotype and virulence properties $[19,20]$. By comparing the open reading frames containing frameshifts in these organisms, we showed that ICDSs could be classified as "common to all strains" or "lineageor strain-specific". The common ICDSs probably correspond to mutations occurring before the divergence of the isolates, whereas lineage- or strain-specific ICDSs correspond to more recently acquired mutations. Thus, ICDS investigation can be used to characterize the molecular scars of evolutionary relationships between organisms and may well provide a unique molecular signature for a particular strain or species, complementary to single nucleotide polymorphism (SNP) and other molecular markers analyses for the characterization of strain variation $[18,21]$. We also show that ICDS formation is not associated with mutation in the promoter region. The present data suggests that promoter extinction is not a major event in the "pseudogenization" process. To experimentally prove that ICDSs comparison is a powerful phylogenomic tool, we analyzed 21 clinical M. tuberculosis isolates for their ICDS content. We showed that the WBeijing lineage differs from the other TB phyla by a lower number of common ICDSs, confirming early divergence with M. tuberculosis s.s strains. ICDS characterization in addition to phylogenetic investigations or typing can be used to select strains or phenotypes for studies of particular phenotypic characters, such as virulence. Indeed, as frameshift acquisition may lead to a loss of function, researchers should consider the possible presence of ICDS before choosing a strain or species for investigating a particular phenotype.

\section{Results \\ Detecting the molecular scars of evolution in $M$. tuberculosis and in $M$. bovis}

Comparative analyses of frameshift-containing genes require the complete genome sequences of closely related organisms. The TB complex, which includes two recently sequenced species and at least 6 accessible strains, is therefore a highly suitable model. We investigated ICDSs in $M$. tuberculosis and in $M$. bovis. The genome sequence of $M$. tuberculosis H37 Rv has been available since 1998 and has recently been re-annotated [22,23]. The genome sequences of $M$. tuberculosis strain CDC1551 and M. bovis have been characterized independently $[18,24]$. The great advantage of studying this model system is that the evolution of these two species and the phylogenetic links between them are well documented [25]. The M. tuberculosis genomes (CDC1551 and H37Rv) have nucleotide 
sequences more than $99.95 \%$ identical to that of $M$. bovis $[18,24]$. The three genomes were screened for the presence of ICDSs. To this end, the genomic sequences of each predicted ICDS [3] were extracted for each strain or species and compared between them. Each common or specific ICDS was then analyzed manually to characterize the molecular event leading to the detected frameshift. The genome of H37RV contains 113 ICDSs, whereas CDC1551 has 137 ICDSs and M. bovis has 134 ICDSs, corresponding to about $2 \%$ of the total coding sequences [3]. These organisms have similar numbers of ICDSs, but the alterations do not always affect the same genes. We therefore investigated whether some of these ICDSs were common to all three organisms. We compared the nucleotide and deduced amino-acid sequences of each frameshiftcontaining open reading frame in the three organisms. We found that 81 of the frameshift-containing genes were common to all three strains (Figure 1A, Table 1), and were identical at the molecular level. The proteins affected by these frameshifts included proteins of unknown function as well as annotated and/or characterized proteins (Table 1 ). The fact that these three mycobacterial genomes were sequenced and assembled independently suggests that these 81 common ICDSs correspond to authentic

A

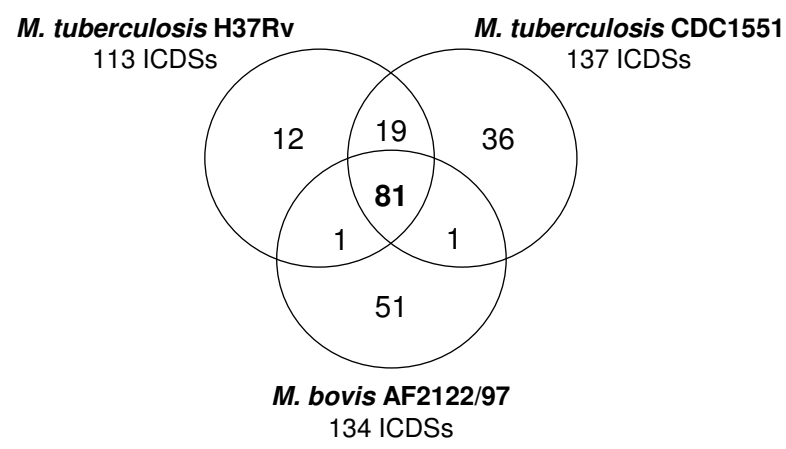

B

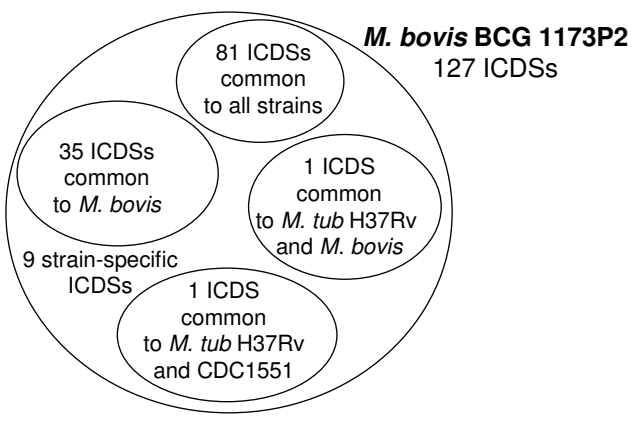

Figure I

A- Schematic representation of the ICDSs common to $M$. tuberculosis H37Rv, CDCI55I and M. bovis AF2 I22/97 or specific to one of these strains. The total number of ICDSs is indicated. B- Schematic representation of the ICDSs of $M$. bovis BCG II73P2 compared to the other analyzed strains. frameshift-containing genes rather than sequencing errors. These results indicate that these 81 ICDSs correspond to frameshifts acquired before the splitting of the M. tuberculosis and M. bovis species (Table 1). Alternatively, the same 81 genetic mutations may result from convergent evolution and hence have occurred independently in all three genomes, a highly unlikely scenario.

The two M. tuberculosis s.s strains were found to have 19 additional common ICDSs, raising their total number to 100 (Figure 1A, Table 2). This suggests that the 19 additional mutations common to these two strains but not to $M$. bovis were acquired post-divergence of $M$. tuberculosis and M. bovis. One ICDS in M. bovis (ICDS0046, Mb1789cMb1790c) was present in $M$. tuberculosis CDC1551 (ICDS0057, MT1807) but not in M. tuberculosis H37Rv $(\operatorname{Rv} 1759 \mathrm{c})$. This mutation (deletion of one $\mathrm{G}$ ) was identical in the M. bovis and M. tuberculosis CDC1551 strains, but an additional mutation was present close to this mutation in the M. bovis genome. One ICDS in M. bovis (ICDS0128, Mb3813-Mb3814) was also present in $M$. tuberculosis H37Rv (ICDS0118, Rv3784-Rv3785) but not in M. tuberculosis CD1551 (MT3893) (Table 2).

The availability of genomic resources for M. tuberculosis is increasing exponentially. This enabled us to investigate the presence or absence of these shared ICDSs in the Haarlem, F11, and C strains, the genomic sequences of which are currently at the assembly stage at the Broad Institute [26]. As the sequence of these genomes is in progress, the total number of frameshift-containing genes in these genomes cannot yet be accurately determined; nonetheless, it is possible to check whether the 81 ICDSs present in M. bovis and in other M. tuberculosis strains are present in these strains. All 81 ICDSs common to all three strains previously tested were also present in Haarlem and F11 strains, while 79 were present in the $\mathrm{C}$ strain (corresponding H37Rv ORFs ICDS0103 and ICDS0105 were fulllength in this strain) (see Additional file 1). Noteworthy, was the identification of additional mutations in the vicinity $(\leq 200 \mathrm{bp}$ ) of the original frameshift (see additional file 1). We next investigated whether the 19 ICDSs common to all M. tuberculosis s.s strains were present in the other clinical isolates. In each case, the ICDSs were also present in the three strains (Haarlem, F11, and C), but accompanied, in some cases, by additional mutations in the flanking region (see Additional file 1). Thus, 98 frameshift-containing genes were found to be conserved in all five M. tuberculosis strains analyzed.

The recently published $M$. bovis BCG genome sequence is of a particular interest in this respect [27]. This strain, which is currently used for vaccination in humans, was derived from $M$. bovis after 13 years of repetitive passages in vitro [28]. A number of genetic differences, such as dele- 
Table I: List of the 8I ICDSs common to M. tuberculosis H37Rv, CDCI55 I, M. bovis AF2 I 22/97 and M. africanum GM04I I82.

M. tub CDC1551 M. bov AF2122197 M. bov BCG 1173 P2 $-M$.

Functional classification

$0002(\mathrm{RvO} 15 \mathrm{Ic} 588$ aa)

0003 (Rv0152c 525 aa)

0007 (Rv0366c 197 aa - Rv0367c 129 aa)

0009 (Rv0393 44I aa)

0010 (Rv0520 II6 aa - Rv052I 101 aa)

0012 (Rv060lc 157 aa)

0014 (Rv0635 I58 aa - Rv0636 /42 aa)

0015 (Rv0636 I42 aa - Rv0637 I66 aa)

0017 (Rv0724A / /2 aa - Rv0725c 30/ aa)

0020 (Rv0865 160 aa)

0021 (Rv0890c 882 aa - Rv089lc 285 aa)

(Rv1034c 130 aa - Rv1035c 228 aa)

024 (Rv1035c $228 a a-R v 1036 c$ / / $2 a a)$

0025 (Rv104lc 287 aa - Rv1042c 135 aa)

0026 (RvI 104229 aa)

0027 (Rv1 $104229 a a)$

0028 (Rvl 105 | 7 I aa)

029 (RvIl I9c 49 aa - RvI I20c $164 a a)$

0030 (RvIl 36 / /3 aa)

0032 (Rvll49 I35 aa-Rvll50 183 aa)

0033 (RvIl63 201 aa - RvIl 64246 aa)

0035 (RvI203c 194 aa - Rvl204c 562 aa)

0036 (Rvl413 17I aa)

0040 (Rv1662 I602 aa - Rv1663 $502 a a)$

0041 (Rv1687c 255 aa)

0043 (Rv1735c 166 aa)

0046 (Rvl878 450 aa)

0047 (Rv1888A 58 aa-Rv1889c $1 / 8$ aa)

0048 (Rv193Ic 259 aa)

$\infty \quad 0049(\operatorname{Rv} 1949$ c $319 a a-\operatorname{Rv} 1950$ c $63 a a)$

0050 (Rv2013 159 aa - Rv2014 196 aa)

005 I (Rv2086 201 aa)

0052 (Rv2086 201 aa - Rv2087 76 aa)

0053 (Rv2087 76 aa)

0054 (Rv2095c 316 aa - Rv2096 332 aa)

058 (Rv232I I82 aa - Rv2322c 22 I ac)

0059 (Rv2325 $282 a a-\operatorname{Rv2326c} 697 a a)$

0060 (Rv233 I I 29 aa)

0061 (Rv2337 372 aa - Rv2338c $3 / 8$ aa)

0062

0004

0006

0109

ICDS
ICDS
ICDS
ICDS

PE family protein

PE family protein

Conserved hypothetical

$\begin{array}{ll}\text { Conserved hypothetical } & \text { Unknown } \\ \text { Dimethylglycine N-methyltransferase } & \text { Intermediary metabolism }\end{array}$

$0012 \quad 0011$

0110

0008

ICDS Dimethylglycine N-methyltransferase

ICDS $^{\circ} \quad$ Two-component sensor kinase

Conserved hypothetical

Conserved hypothetical

Conserved hypothetical

Molybdopterin biosynthesis protein Intermediary metabolism

Transcriptional regulator

Transposase

Transposase

Transposase

Esterase

Esterase

Para-nitrobenzyl esterase

Conserved hypothetical

Enoyl-CoA

Transposase

Nitrate reductase Narl-J

Conserved hypothetical

Conserved hypothetical

Polyketide synthase Pks 8117

ATP binding protein, $A B C$ transporter

Malic acid transport protein

Glutamine synthetase $\mathrm{Gln} A$

Conserved hypothetical

Conserved hypothetical

Conserved hypothetical

Transposase

Conserved hypothetical

Conserved hypothetical

Conserved hypothetical

$\begin{array}{ll}\text { Conserved hypothetical } & \text { Unknown } \\ \text { Ornithine aminotransferase RocDI } & \text { Intermediary metabolism }\end{array}$

Conserved hypothetical

Hypothetical

Conserved hypothetical

Hydrogenase nickel incorporation protein HypB Intermediary metabolism

0063 (Rv2877c 287 aa - Rv2878c 173 aa)

ICDS

Conserved hypothetical

PE/PPE

PE/PPE

Intermediary metabolism

Regulation

Unknown

Regulation

S/phage

IS/phage

Intermediary metabolism

Intermediary metabolism

Intermediary metabolism

Unknown

Lipid metabolism

IS/phage

Intermediary metabolism

Unknown

Lipid metabolism

Cell wall, process

Cell wall, process

Intermediary metabolism

Unknown

Unknown

Unknown

IS/phage

Unknown

Unknown

Unknown

Unknown

0068

0076

Unknown

$\begin{array}{ll}0065 & 0076 \\ 0099 & 0077\end{array}$


Table I: List of the 8 I ICDSs common to M. tuberculosis H37Rv, CDCI55 I, M. bovis AF2 I 22/97 and M. africanum GM04I I82. (Continued)

\begin{tabular}{|c|c|c|c|c|c|c|}
\hline 0065 (Rv2943A I77 aa - Rv2944 239 aa) & 0083 & 0066 & 0079 & ICDS & Transposase & IS/phage \\
\hline $0068(\operatorname{Rv} 3128 c 338 a a)$ & 0088 & 0068 & 0082 & ICDS & Conserved hypothetical & Unknown \\
\hline 0069 (Rv3152 410 aa - Rv3153 2II aa) & 0089 & 0100 & 0121 & ICDS & NADH dehydrogenase I & Intermediary metabolism \\
\hline $0070(\mathrm{Rv} 3 \mathrm{I} 72 \mathrm{c} 160 \mathrm{aa})$ & 0090 & 0069 & 0083 & ICDS & Conserved hypothetical & Unknown \\
\hline $0071(\operatorname{Rv3200c} 355 a a)$ & 0091 & 0101 & 0122 & ICDS & Hypothetical & Unknown \\
\hline $0075(\operatorname{Rv3349c} 246 a a)$ & 0100 & 0102 & 0085 & $\mathrm{ICDS}^{\circ}$ & Transposase & IS/phage \\
\hline $0076(\operatorname{Rv} 335$ Ic $264 a a-\operatorname{Rv} 3352 c / 23 a a)$ & 0101 & 0071 & 0088 & ICDS & Oxidoreductase & Intermediary metabolism \\
\hline $0077(\operatorname{Rv3352c} 123 a a-\operatorname{Rv} 3353 c 86 a a)$ & 0102 & 0072 & 0089 & ICDS & Oxidoreductase & Intermediary metabolism \\
\hline $0079(\operatorname{Rv} 3419 c 344 a a)$ & 0104 & 0135 & 0131 & ICDS & O-sialoglycoprotein endopeptidase & Intermediary metabolism \\
\hline $0080(\operatorname{Rv} 3420 \mathrm{c} 158 a a-\operatorname{Rv} 342 \mathrm{Ic} 21 / \mathrm{aa})$ & 0105 & 0104 & 0092 & $\mathrm{ICDS}^{\circ}$ & Conserved hypothetical & Unknown \\
\hline 0083 & 0108 & 0107 & 0095 & ICDS & Transposase & IS/phage \\
\hline 0087 (Rv374lc $224 a a-R v 3742 c$ 13I aa) & 0114 & 0078 & 0099 & $\mathrm{ICDS}^{\circ}$ & Aromatic-ring hydroxylase & Intermediary metabolism \\
\hline 0088 (Rv3770A 61 aa - Rv3770B 64 aa) & 0115 & 0079 & 0100 & $\mathrm{ICDS}^{\circ}$ & Transposase & IS/phage \\
\hline $0089(\operatorname{Rv} 3844$ I64 aa - Rv3845 I20 aa) & 0116 & 0136 & 0132 & ICDS & Transposase & IS/phage \\
\hline $0090(\operatorname{Rv} 3866283 a a-R v 3867$ 183aa) & 0117 & 0109 & 0123 & ICDS & Conserved hypothetical & Unknown \\
\hline $0091(\operatorname{Rv3880c} / 15 a a-\operatorname{Rv} 388 \mid 460 a a)$ & 0118 & 0137 & 0133 & ICDS & Conserved hypothetical & Unknown \\
\hline $0095(\operatorname{Rv3900c} 3 / /$ aa $)$ & 0122 & 0083 & 0124 & ICDS & Conserved hypothetical & Unknown \\
\hline 0097 (Rv3913 $335 a a-\operatorname{Rv3914} / / 16 a a)$ & 0123 & 0111 & 0001 & ICDS & Thioredoxin reductase & Intermediary metabolism \\
\hline 0098 & 0124 & 0032 & 0035 & ICDS & Conserved hypothetical & Unknown \\
\hline $0099(\operatorname{Rv} 3386234 a a-\operatorname{Rv3387} 225 a a)$ & 0125 & 0103 & 0090 & ICDS & Transposase & IS/phage \\
\hline 0100 (Rv0342 $640 a a-R v 0343493 a a)$ & 0126 & 0009 & 0125 & ICDS & Isoniazid inductible gene protein & Cell wall, process \\
\hline 0101 (Rv0763c $69 a a-R v 0764 c 45 I a a)$ & 0127 & 0084 & 0126 & ICDS & Cytochrome P450 & Intermediary metabolism \\
\hline $0103(\mathrm{Rv0449c} 439 \mathrm{aa})$ & 0129 & 0113 & 0010 & ICDS & Conserved hypothetical & Unknown \\
\hline 0104 (Rv047lc $162 \mathrm{aa})$ & 0130 & 0114 & 0011 & ICDS & Hypothetical & Unknown \\
\hline 0105 (Rv0859 403 aa - Rv0860 $720 a a)$ & 0131 & 0115 & 0024 & $\mathrm{ICDS}^{\circ}$ & $\begin{array}{l}\text { Acyl-CoA thiolase FadA and dehydrogenase } \\
\text { FadB }\end{array}$ & Lipid metabolism \\
\hline 0106 (Rv0880 I 43 aa - Rv088I $288 a a)$ & 0132 & 0116 & 0025 & $\mathrm{ICDS}^{\circ}$ & Transcriptional regulator & Information pathway \\
\hline 0107 (Rv0997 I43 aa) & 0133 & 0117 & 0029 & ICDS & Hypothetical & Unknown \\
\hline $0108(\operatorname{Rv} 104$ Ic $287 a a-R v 1042 c / 35 a a)$ & 0134 & 0118 & 0033 & $\mathrm{ICDS}^{\circ}$ & Transposase & IS/phage \\
\hline 0109 (Rvll04 $229 a a-R v 1105$ I7I aa) & 0135 & 0119 & 0038 & ICDS & Para-nitrobenzyl esterase & Intermediary metabolism \\
\hline 0110 (RvI22I $257 a a-\operatorname{RvI} 222154 a a)$ & 0136 & 0120 & 0044 & ICDS & Alternative sigma factor SigE & Information pathway \\
\hline 011 (Rv1752c /49 aa) & 0137 & 0121 & 0051 & ICDS & Conserved hypothetical & Unknown \\
\hline $0112(\operatorname{Rv} 196 \mid 164 a a)$ & 0138 & 0122 & 0060 & ICDS & Hypothetical & Unknown \\
\hline $0113(\operatorname{Rv} 2309 \mathrm{c} 15 / a a)$ & 0139 & 0123 & 0066 & ICDS & Integrase & Information pathway \\
\hline $0114(R v 2420 c 127 a a-R v 242$ Ic $21 /$ aa $)$ & 0140 & 0124 & 0070 & ICDS & nicotinate-nucleotide adenyly|transferase $\mathrm{NadD}$ & Intermediary metabolism \\
\hline 0115 (Rv2732c $205 a a-R v 2733 c 5 / 2 a a)$ & 0141 & 0125 & 0073 & ICDS & Conserved hypothetical & Unknown \\
\hline 0116 (Rv2922A $94 a a-\operatorname{Rv2923c/37aa)}$ & 0142 & 0126 & 0078 & ICDS & Acylphosphatase AcyP & Intermediary metabolism \\
\hline 0117 (Rv3774 $274 a a-\operatorname{Rv3775} 274 a a)$ & 0143 & 0127 & 0101 & $\mathrm{ICDS}^{\circ}$ & Enoyl-CoA hydratase EchA2I and lipase LipE & Lipid metabolism \\
\hline 0119 (Rv2599 I43 aa - Rv2600 I33 aa) & 0144 & 0098 & 0134 & $\mathrm{ICDS}^{\circ}$ & Conserved hypothetical & Unknown \\
\hline
\end{tabular}

ICDS number (variable, according to the strain), the size of the predicted protein and its putative function are indicated. The corresponding ORF numbers in $M$. tuberculosis $\mathrm{H} 37 \mathrm{Rv}$ are indicated in brackets. "'" indicates ICDSs containing additional mutations with respect to M. tuberculosis H37Rv, CDCI55I and M. bovis AF2I 22/97. 
Table 2: List of the 19 ICDSs common to $M$. tuberculosis H37Rv and CDCI55I, the ICDSs common to M. tuberculosis H37Rv and M. bovis AF2I $22 / 97$ and the ICDSs common to M. tuberculosis CDC155I and M. bovis AF2122/97

\begin{tabular}{|c|c|c|c|c|c|c|}
\hline M. tub H37Rv & M. tub CDCI55I & M. bovis AF2 I 22/97 & M. tub 210 & M. africanum & Putative function & Functional classification \\
\hline 0001 (Rv0095c I36 aa) & 0003 & Mb0098c 260 aa & ICDS & Not Found & Conserved hypothetical & Unknown \\
\hline 0005 (Rv0325 $74 a a-\operatorname{Rv0326~/5/~aa)~}$ & 0010 & Mb0333229 aa & $\mathrm{FL}$ & $\mathrm{FL}$ & Hypothetical & Unknown \\
\hline 0011 (Rv0590 $275 a a-\operatorname{Rv0590A~} 84 a a)$ & 0016 & Mb0605 $343 a a$ & $\mathrm{FL}$ & $\mathrm{FL}$ & MCE-family protein & Virulence, detox, adapt \\
\hline 0013 (Rv06I8 23I aa-Rv06I9 I8I aa) & 0018 & Mb0635 $394 a a$ & ICDS & $\mathrm{FL}$ & Galactose-I-phosphate uridylyltransferase & Intermediary metabolism \\
\hline 0022 (Rv0924c $428 a a-R v 0925 c 245 a a)$ & 0028 & Mb0948c $684 a a$ & ICDS & ICDS & Manganese transport protein $\mathrm{MntH}$ & Cell wall, process \\
\hline 003 I (RvII45 $303 a a-R v 1$ I46 $470 a a)$ & 0040 & MbII77 $78 I a a$ & $\mathrm{FL}$ & $\mathrm{FL}$ & Transmembrane transport protein MmpLI3 & Cell wall, process \\
\hline 0037 (Rv1503c I82 aa-Rv1504c $199 a a)$ & 0048 & MbI542c $382 a a$ & ICDS & $\mathrm{FL}$ & Conserved hypothetical & Unknown \\
\hline 0038 (Rv1549 I75 aa-Rv1550 57I aa) & 0051 & MbI576 $647 a a$ & ICDS & $\mathrm{FL}$ & Fatty-acid-coA ligase FadDI I & Lipid metabolism \\
\hline $0039(\operatorname{Rv} 1553-247 a a-R v / 554$ I26aa) & 0052 & MbI579 $374 a a$ & $\mathrm{ICDS}^{\circ}$ & $\mathrm{ICDS}^{\circ}$ & Fumarate reductase & Intermediary metabolism \\
\hline $0045(\operatorname{Rv} 179259$ aa) & 0058 & Mbl82098 aa & ICDS & $\mathrm{FL}$ & ESAT-6-like protein EsxM & Cell wall, process \\
\hline 0055 (Rv2227 $233 a a)$ & 0072 & Mb2252 I24aa & ICDS & $\mathrm{FL}$ & Conserved hypothetical & Unknown \\
\hline 0066 (Rv2946c $16 / 6$ aa - Rv2947 $496 a a)$ & 0084 & Mb297 Ic $21 / 2$ aa & $\mathrm{FL}$ & $\mathrm{FL}$ & Polyketide synthase Pks I5/I & Lipid metabolism \\
\hline 0067 (Rv2974c 470 aa - Rv2975c $84 a a)$ & 0085 & Mb2999c 553 aa & $\mathrm{FL}$ & $\mathrm{FL}$ & Conserved hypothetical & Unknown \\
\hline $0072(\operatorname{Rv} 3233 c / 96$ aa - Rv3234c 27I aa) & 0092 & Mb3262c 469 aa & FL & $\mathrm{FL}$ & Conserved hypothetical & Unknown \\
\hline $0073(\operatorname{Rv3337} / 28 a a-\operatorname{Rv} 3338$ 2/4aa) & 0094 & Mb3370 297 aa & ICDS & $\mathrm{FL}$ & Conserved hypothetical & Unknown \\
\hline $0078(\operatorname{Rv} 3373213 a a-\operatorname{Rv} 337482 a a)$ & 0103 & Mb3408 296 aa & ICDS & $\mathrm{FL}$ & Enoyl-CoA hydratase EchAI8 & Lipid metabolism \\
\hline $0085(\operatorname{Rv} 3725309 a a)$ & 0112 & Mb3752 333 aa & $\mathrm{FL}$ & $\mathrm{FL}$ & Oxidoreductase & Intermediary metabolism \\
\hline $0086(\operatorname{Rv3738c} 3 / 5 a a-\operatorname{Rv} 3739 c 77 a a)$ & 0113 & Not determined & ICDS & ICDS & PPE family protein & $\mathrm{PE} / \mathrm{PPE}$ \\
\hline $0094(\operatorname{Rv3897c} 210 a a-\operatorname{Rv3898c} / 10 a a)$ & 0121 & Mb3927c 329 aa & $\mathrm{FL}$ & $\mathrm{FL}$ & Conserved hypothetical & Unknown \\
\hline M. tub H37Rv & M. bovis AF2 I 22/97 & M. tub CDCI55I & M. tub 210 & M. africanum & Putative function & Functional classification \\
\hline 0118 (Rv3784 $326 a a-\operatorname{Rv3785} 357 a a)$ & 0128 & MT3893 7/2 aa & NT & ICDS & NAD-dependent epimerase/dehydratase & Information pathway \\
\hline M. tub CDCI55I & M. bovis AF2 I 22/97 & M. tub H37Rv & M. tub 210 & M. africanum & Putative function & Functional classification \\
\hline 0057 (MTI806 820 aa - MTI80794 aa) & 0046 & Rv1759c $9 / 4$ aa & NT & NT & PE_PGRS family protein & PE/PPE \\
\hline
\end{tabular}

List of the 19 ICDSs common to M. tuberculosis H37Rv and CDCI55I, the ICDSs common to M. tuberculosis H37Rv and M. bovis AF2I22/97 and the ICDSs common to M. tuberculosis CDCI55I and M. bovis AF2 I22/97. ICDS number (variable, according to strain), the size of the predicted protein and its putative function are indicated. The genes that do not contain a frameshift in either $M$. tuberculosis strain 210 and in M. africanum and that correspond to a full-length ORF are noted "FL". "NT", not tested. 
tions and duplications had already been identified in the BCG strain [29,30], but large amounts of additional information have now been obtained from its genome sequence. According to our investigation, M. bovis BCG 1173 P 2 contains 127 ICDSs in total, 9 of which are strainspecific (Figure 1B). The 81 ICDSs common to the 3 other isolates are also present in this strain (Table 1) and 35 ICDSs are common to the M. bovis strain. We detected frameshift-containing genes in M. bovis AF2122/97 that corresponded to full-length ORFs in $M$. bovis BCG $1173 \mathrm{P} 2$, suggesting that this $M$. bovis strain is not the direct progenitor of the BCG vaccine (see Additional file 2).

\section{Strain-specific ICDSs reflect newly acquired mutations and are a useful phylogenetic tool}

Eighty-one ICDSs were common to all three strains, but some were specific to one strain only: 12 for M. tuberculosis H37Rv (see Additional file 3), 36 for CDC1551 (see Additional file 4) and 51 for M. bovis (see Additional file 2, Figure $1 \mathrm{~A}$ ). The proportion of ICDSs that were strain-specific was highly variable. These ICDSs accounted for $10 \%$ of all ICDSs in H37Rv, 26\% in CDC1551 and 38\% in M. bovis. The much larger proportion of strain-specific ICDSs in CDC1551 than in H37Rv strain is surprising, and we currently have no reasonable explanation for this phenomenon. A plausible hypothesis is that the genome sequence of CDC1551 strain has not been re-sequenced like the H37Rv genome sequence [22,28]. Strain-specific frameshift-containing genes most likely correspond to mutations acquired after the divergence of these strains. Like the common ICDSs, these events affected genes from several classes, including "unknown or hypothetical ORFs", "intermediary metabolism" and "cell wall, process" (Additional files 2, 3 and 4). As stated above, few of these strain-specific ICDSs may correspond to errors introduced during the sequencing procedure [4,11], but such errors would nonetheless have only a slight effect on the overall outcome of the comparative analysis.

This study shows that the genome sequence of $M$. tuberculosis contains ICDSs that have been acquired during the evolution of this species. The pool of ICDSs can be classified into ICDSs common to a set of strains or species and ICDSs specific to a particular strain-lineage or strain, revealing genetic differences between strains or species.

\section{Using ICDS comparisons to type W-Beijing strains and other $M$. tuberculosis lineages}

$\mathrm{W}$-Beijing is a lineage of $M$. tuberculosis that has attracted considerable attention. Indeed, strains of this lineage have been implicated in severe outbreaks and have been shown to have different genetic and phenotypic properties $[20,21,31]$. The genome of a strain of the W-Beijing family (strain 210) is currently sequenced but not yet fully assembled; nevertheless it can be consulted in homology searches. Consequently the total number of frameshiftcontaining genes in this species and the full characterization of specific ICDSs remain elusive. It is however possible to screen for the presence of ICDSs in this strain.

We first investigated whether the 81 frameshift-containing genes common to all strains were also present in the genome of strain 210. All 81 of these genes also contained the same frameshift in strain 210, in agreement with the data described above. This suggests that these 81 frameshift mutations were acquired before the divergence of strain 210 from these other strains. We then investigated the 19 genes containing frameshifts common to the five strains of M. tuberculosis (H37Rv, CDC1551, Haarlem, F11, C) but not to $M$. bovis. We found that eight of these 19 genes contain no frameshift in strain 210, and hence corresponded to full-length ORFs (Table 2). Three genes contained frameshifts corresponding to those observed in strains CDC1551, H37Rv, Haarlem, F11 and C, but also contained additional mutations in the corresponding flanks ( $\leq 200 \mathrm{bp}$ ) of the original frameshift (Table 2$)$. The remaining 11 ICDSs corresponded to frameshift-containing genes common to all six $\mathrm{TB}$ strains examined (CDC1551, H37Rv, Haarlem, F11, C, 210) and the events were identical at the molecular level. Thus, the 19 frameshift-containing genes in the two TB strains (CDC1551 and H37Rv) displayed polymorphism in strain 210 and 11 of these identified ICDSs were common to all six TB strains examined. Some of these ICDSs display no further mutation (the gene contains the frameshift alone), whereas others have acquired additional mutations, contributing to the "pseudogenization" process (data not shown).

We then investigated the eight ICDSs showing polymorphism in M. tuberculosis in 21 strains of the W-Beijing lineage from several phylogenetic groups (Table 3 ). The eight loci were amplified by PCR, sequenced and the nucleotide sequence was compared with that of strains 210 and $\mathrm{H} 37 \mathrm{Rv}$. In all W-Beijing strains tested, the eight genes were full-length, with sequences $100 \%$ identical to that in strain 210, excepted for the ICDS0085 where a non-disruptive SNP is present in the region. The W-Beijing lineage is therefore a genetically homogeneous group with fewer ICDSs in common with other TB strains.

To extend our analysis, we investigate the M. africanum strain, which is currently sequenced at the Sanger centre. Similarly to $M$. tuberculosis 210 strain, the M. africanum genome is still at the assembly step, but can be nevertheless consulted on line. We investigated whether the 81 frameshift containing genes common to all strains tested were also present in the M. africanum strain (Table 1). All 81 of these genes also contained a frameshift in $M$. africa- 
Table 3: Analysis in 2I W-Beijing isolates of the 8 ICDSs of H37Rv strain corresponding to full-length ORFs in W-Beijing strain 210.

\begin{tabular}{|c|c|c|c|c|c|c|c|c|c|c|}
\hline & Finger print & Tracking Number & ICDS 0005 & ICDS 0011 & ICDS 003 I & ICDS 0066 & ICDS 0067 & ICDS 0072 & ICDS 0085 & ICDS 0094 \\
\hline \multirow[t]{12}{*}{ W-Beijing } & W & 10648 & $\mathrm{FL}$ & $\mathrm{FL}$ & $\mathrm{FL}$ & $\mathrm{FL}$ & $\mathrm{FL}$ & $\mathrm{FL}$ & NT & $\mathrm{FL}$ \\
\hline & $\mathbf{W}$ & 565 & $\mathrm{FL}$ & $\mathrm{FL}$ & $\mathrm{FL}$ & $\mathrm{FL}$ & $\mathrm{FL}$ & $\mathrm{FL}$ & $\mathrm{FL}^{*}$ & $\mathrm{FL}$ \\
\hline & W4 & 10775 & $\mathrm{FL}$ & $\mathrm{FL}$ & $\mathrm{FL}$ & $\mathrm{FL}$ & $\mathrm{FL}$ & $\mathrm{FL}$ & $\mathrm{FL}^{*}$ & $\mathrm{FL}$ \\
\hline & WI4 & 3617 & $\mathrm{FL}$ & $\mathrm{FL}$ & $\mathrm{FL}$ & $\mathrm{FL}$ & $\mathrm{FL}$ & $\mathrm{FL}$ & $\mathrm{FL}^{*}$ & $\mathrm{FL}$ \\
\hline & W26 & 10270 & $\mathrm{FL}$ & $\mathrm{FL}$ & $\mathrm{FL}$ & $\mathrm{FL}$ & $\mathrm{FL}$ & $\mathrm{FL}$ & $\mathrm{FL}^{*}$ & $\mathrm{FL}$ \\
\hline & W69 & 5418 & $\mathrm{FL}$ & $\mathrm{FL}$ & $\mathrm{FL}$ & $\mathrm{FL}$ & $\mathrm{FL}$ & $\mathrm{FL}$ & $\mathrm{FL}^{*}$ & $\mathrm{FL}$ \\
\hline & W88 & 7052 & $\mathrm{FL}$ & $\mathrm{FL}$ & $\mathrm{FL}$ & $\mathrm{FL}$ & $\mathrm{FL}$ & $\mathrm{FL}$ & $\mathrm{FL}^{*}$ & $\mathrm{FL}$ \\
\hline & W130 & 6707 & $\mathrm{FL}$ & $\mathrm{FL}$ & $\mathrm{FL}$ & $\mathrm{FL}$ & $\mathrm{FL}$ & $\mathrm{FL}$ & $\mathrm{FL}^{*}$ & $\mathrm{FL}$ \\
\hline & WI48 & $856 I$ & $\mathrm{FL}$ & $\mathrm{FL}$ & $\mathrm{FL}$ & $\mathrm{FL}$ & $\mathrm{FL}$ & $\mathrm{FL}^{*}$ & $\mathrm{FL}^{*}$ & $\mathrm{FL}$ \\
\hline & W 183 & 7657 & $\mathrm{FL}$ & $\mathrm{FL}$ & $\mathrm{FL}$ & $\mathrm{FL}$ & $\mathrm{FL}$ & $\mathrm{FL}$ & NT & $\mathrm{FL}$ \\
\hline & W2I5 & 8963 & $\mathrm{FL}$ & $\mathrm{FL}$ & $\mathrm{FL}$ & $\mathrm{FL}$ & $\mathrm{FL}$ & $\mathrm{FL}$ & $\mathrm{FL}^{*}$ & $\mathrm{FL}$ \\
\hline & W342 & 10644 & $\mathrm{FL}$ & $\mathrm{FL}$ & $\mathrm{FL}$ & $\mathrm{FL}$ & $\mathrm{FL}$ & $\mathrm{FL}$ & $\mathrm{FL}^{*}$ & $\mathrm{FL}$ \\
\hline \multirow[t]{7}{*}{ Ancestral W-Beijing } & NI7 & 3046 & $\mathrm{FL}$ & $\mathrm{FL}$ & $\mathrm{FL}$ & $\mathrm{FL}$ & $\mathrm{FL}$ & $\mathrm{FL}$ & $\mathrm{FL}^{*}$ & $\mathrm{FL}$ \\
\hline & LB & 8128 & $\mathrm{FL}$ & $\mathrm{FL}$ & $\mathrm{FL}$ & $\mathrm{FL}$ & $\mathrm{FL}$ & $\mathrm{FL}$ & $\mathrm{FL}^{*}$ & $\mathrm{FL}$ \\
\hline & $\mathbf{A R}$ & 12360 & $\mathrm{FL}$ & $\mathrm{FL}$ & $\mathrm{FL}$ & $\mathrm{FL}$ & $\mathrm{FL}$ & $\mathrm{FL}$ & $\mathrm{FL}^{*}$ & $\mathrm{FL}$ \\
\hline & $\mathbf{A M}$ & 4948 & $\mathrm{FL}$ & $\mathrm{FL}$ & $\mathrm{FL}$ & $\mathrm{FL}$ & $\mathrm{FL}$ & $\mathrm{FL}$ & $\mathrm{FL}^{*}$ & $\mathrm{FL}$ \\
\hline & HE7 & 13454 & $\mathrm{FL}$ & $\mathrm{FL}$ & $\mathrm{FL}$ & $\mathrm{FL}$ & $\mathrm{FL}$ & $\mathrm{FL}$ & $\mathrm{FL}^{*}$ & $\mathrm{FL}$ \\
\hline & HI & 5116 & $\mathrm{FL}$ & $\mathrm{FL}$ & $\mathrm{FL}$ & $\mathrm{FL}$ & $\mathrm{FL}$ & $\mathrm{FL}$ & $\mathrm{FL}^{*}$ & $\mathrm{FL}$ \\
\hline & KY & 10583 & $\mathrm{FL}$ & $\mathrm{FL}$ & NT & $\mathrm{FL}$ & $\mathrm{FL}$ & $\mathrm{FL}$ & $\mathrm{FL}^{*}$ & $\mathrm{FL}$ \\
\hline AF/H37 lineage & H37Rv & ATCC256I 8 & ICDS & ICDS & ICDS & ICDS & ICDS & ICDS & ICDS & ICDS \\
\hline M. bovis AF2 I 22/97 & & & $\mathrm{FL}$ & $\mathrm{FL}$ & $\mathrm{FL}$ & $\mathrm{FL}$ & $\mathrm{FL}$ & $\mathrm{FL}$ & $\mathrm{FL}$ & $\mathrm{FL}$ \\
\hline
\end{tabular}

M. tuberculosis isolates from various lineages for which chromosomal DNA was used as a template for PCR amplification of the selected locus. "FL" indicates the presence of a fulllength ORF identical to that in the M. tuberculosis 210 strain, "*" indicates an additional mutation acquired in these isolates with respect to M. tuberculosis H37Rv, "NT", not tested. 


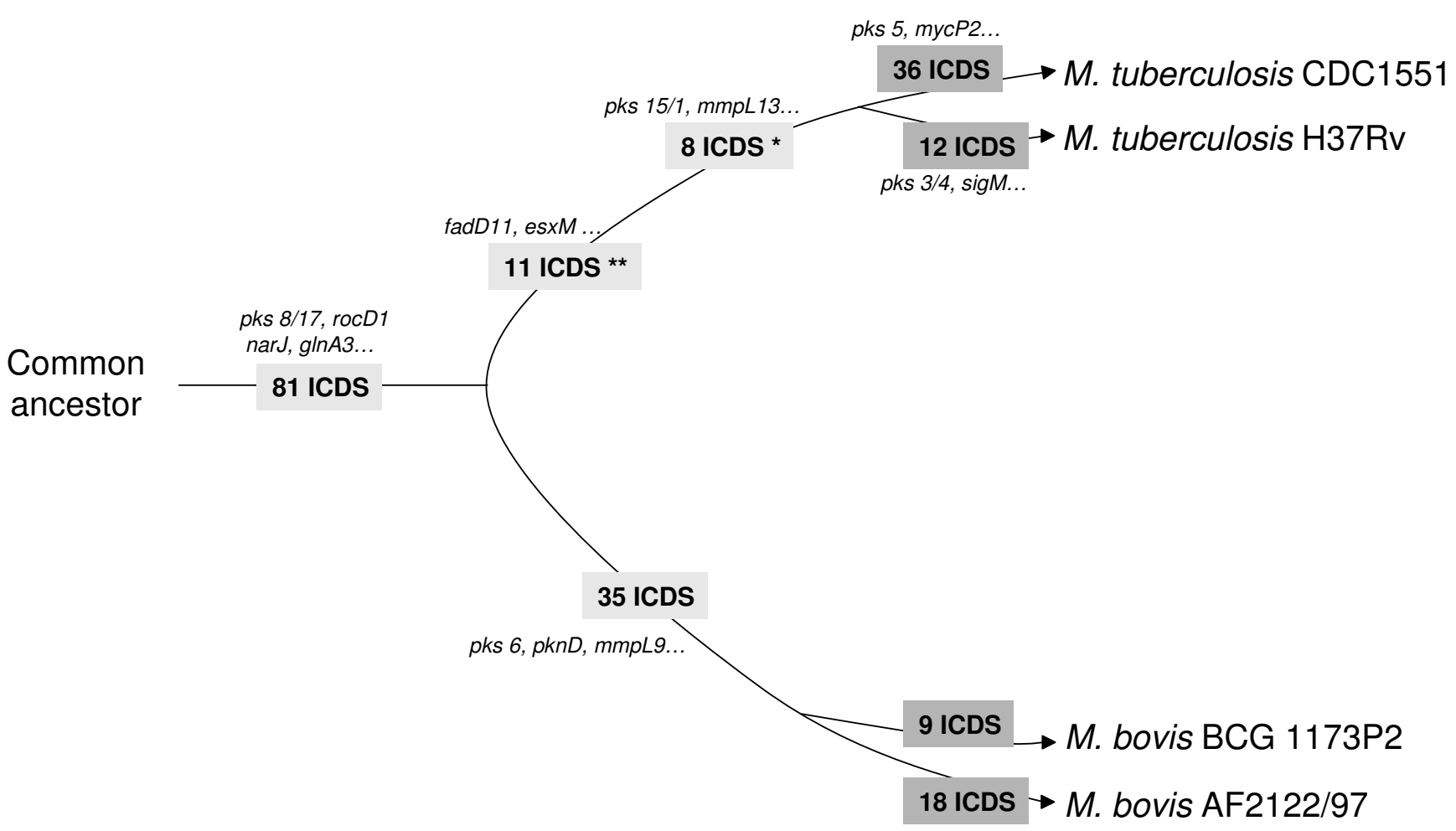

Figure 2

Hypothetical phylogenetic links assessed by comparative analyses of ICDSs. In this schematic representation, the common ancestor gave rise to several branches of strains of the TB complex. Eighty-one frameshifts were acquired during the common evolution of $M$. bovis and $M$. tuberculosis. Since the separation of these species, $M$. bovis has acquired $5 \mathrm{I}$ frameshifts, while the branch leading to $M$. tuberculosis isolates has acquired 19 new frameshifts. Since separation of the isolates, M. tuberculosis H37Rv has acquired 12 new frameshifts and CDCI55I 36 new frameshifts. Common and unique ICDSs are shown in dark and light gray, respectively. "*" these 8 ICDSs correspond to full-length ORF in M. tuberculosis 210 and in M. africanum GM04I I82. "**" 7 out of these II ICDSs correspond to full-length ORF in M. africanum GM04I I 82 (Table 2).

num, which suggests that these mutations were acquired before the divergence of the M. tuberculosis complex. We then investigated the 19 genes containing frameshift common to the $5 \mathrm{M}$. tuberculosis strains (CDC1551, H37Rv, Haarlem, F11, C). We found that 15 out of these 19 genes were deprived of the frameshift in M. africanum and corresponded to full-length ORFs in this strain (Table 2). Eight out of these 15 genes match the wild-type ORFs identified in M. tuberculosis strain 210 and other strains of the W-Beijing lineage. In conclusion, the genome of $M$. africanum contains fewer ICDSs in common with the other TB isolates (CDC1551, H37Rv, Haarlem, F11, C) than with the $\mathrm{W}$-Beijing strain and seems genetically closer to this lineage.

\section{ICDS formation is not correlated with mutation in the promoter region}

It has been suggested that pseudogene formation is associated with mutations in the upstream untranslated region, abolishing pseudogene expression to prevent a loss of metabolic function [32]. Once turned off, the gene continues to accumulate mutations, leading to complete pseudogene formation. ICDSs are not pseudogenes in the strict sense of the word. Indeed, the ORF is split into only two or three unframed fragments and can, in theory, revert to a wild-type allele. ICDSs are therefore considered to be ORFs undergoing "pseudogenization" rather than pseudogenes per se. Strain-specific ICDSs are, by definition, genes that are mutated in one strain, but not in another. We therefore investigated whether ICDS formation was correlated with mutation in the promoter region. All the intergenic regions (99) located upstream from strain-specific ICDSs of M. tuberculosis H37Rv, CDC1551 and $M$. bovis were compared with the corresponding region in the two strains having a wild-type gene. We used as a control the promoter region of randomly selected genes that are full-length in these 3 strains. We compared the level of differences observed in the promoter regions of genes full-length or containing frameshift. Nucleotide differences were observed in $27 \%$ of the upstream region 
of genes containing frameshift (see Additional file 5A), while $20 \%$ was observed in the case of the full-length genes (see Additional file 5B), which is not statistically significant using the chi square test. In all but 6 cases for ICDS and 2 cases for full-length genes, the difference in the upstream region was limited to one or two SNPs.

We therefore conclude that ICDS formation is not correlated with mutation in the untranslated upstream region and suggest that either promoter mutations do not play a major role in pseudogene formation in the M. tuberculosis complex or that "pseudogenization" is recent.

\section{Discussion}

The presence of frameshift-containing genes in bacterial genomes is well documented [1-3,33]. A few species can bypass such frameshifts, but most do not, generally resulting in a loss of function.

We show here that ICDSs can be classified as "common to all strains" or "strain-specific". The ICDSs common to all strains probably correspond to mutations acquired before the divergence of the strains, whereas strain-specific ICDSs correspond to those acquired subsequently (Figure 2). Mutations acquired after the speciation of $M$. tuberculosis from M. bovis were also detected. We identified 19 ICDSs common to the five M. tuberculosis strains (H37Rv, CDC1551, Haarlem, F11 and C) but not to M. bovis, about one-fifth of ICDSs common to all strains. Comparative analyses of ICDSs help to characterize the phylogenetic relationships between highly related strains and species (Figure 2) and could be applied to any bacterial species for which several genome sequences are available. In few cases, ICDSs may correspond to fusion/fission of orthologous genes in other genomes. The detection of this kind of events is due to the method of identification of ICDS but remains however a minor inconvenience [3]. It is however possible that a low percentage of specific ICDSs does correspond to sequencing errors, inducing thus artifactual phylogenetic relationships. Researchers should resequence these regions before assuming that the ICDS corresponds to a frameshift acquisition. Several studies have compared the genome sequences of $M$. tuberculosis CDC1551 and H37Rv, using high-resolution genomics techniques [18]. This has led to the definition of regions containing large-sequence polymorphisms (LSPs, greater than $10 \mathrm{bp}$ ) and single nucleotide polymorphisms (SNPs). The SNPs have been investigated in more detail in various clinical isolates, to draw up a global phylogeny of M. tuberculosis [17]. Other molecular methods, such as analyses of the deleted regions (deligotyping), variable numbers of tandem repeats (VNTR), mycobacterial interspersed repetitive unit (MIRU) and spoligotyping, have helped to unravel global genomic sequence diversity in this species [34-36]. These techniques are highly useful for epidemiological studies, but as far provide little information pertaining to genetic differences in terms of putative function. In contrast, studies of regions of deletion (RD) have proved useful for both global phylogeny and study of a loss of phenotype in both $M$. tuberculosis and in $M$. ulcerans $[25,30,37]$.

Frameshift acquisition generally leads to a loss of function, as shown in a number of published studies. Loss-offunction associated with the presence of a frameshift has been reported in both $M$. tuberculosis and M. bovis. For instance, ICDS0066 in M. tuberculosis H37Rv corresponds to a frameshift-containing gene encoding a polyketide synthase ( $p k s 1)$. This pks1 gene also contains a frameshift in M. tuberculosis CDC1551, resulting in two different ORFs: pks 1 and pks15. In contrast, $M$. bovis and M. leprae carry a full-length functional pks1 gene [38]. The pks15/1 gene is now frequently used as a marker in epidemiological studies $[39,40]$ and, interestingly, the pks gene contains no frameshift in the W-Beijing strains of $M$. tuberculosis [40], resulting in phenolglycolipid production in most cases [41]. Our analysis shows that the pks gene of M. africanum is also full-length suggesting that this species produces PGL. This observation suggests that these early strains are more closely related to $M$. bovis or to the last ancestor than other M. tuberculosis strains. Similarly, ICDS0067 in $M$. bovis corresponds to a putative frameshift-containing glycosyltransferase gene. The ortholog of this gene has no frameshift in the two strains of M. tuberculosis (Rv2958c and MT3034). Functional complementation of M. bovis BCG with the Rv2958c gene from M. tuberculosis leads to the accumulation of a new metabolite, the diglycosylated phenolglycolipid [42]. Some frameshift-containing genes have been studied experimentally in M. tuberculosis, without considering the possibility that these ORFs may well contain frameshift $[43,44]$. Mutation by homologous recombination has been achieved at the $m n t H$ and $m m p L 13$ loci. In both cases, no detectable phenotype was associated with the mutation. Our data indicate that MmpL13 function should be investigated in a W-Beijing strain or in M. africanum. Another example that has not yet been studied is the pks3 and pks4 genes of $M$. tuberculosis H37Rv, which constitute a single ORF in CDC1551 and in M. bovis. This suggests that - like the pks 1 and pks 15 genes, which are pseudogenes in M. tuberculosis - the pks 3 and pks 4 genes are probably not functional in the H37Rv strain. It would therefore be pointless to investigate function in the $\mathrm{H} 37 \mathrm{Rv}$ strain by creating mutants in pks 3 and pks 4 genes or by expressing constructs encoding the corresponding polypeptides. These examples from previous publications illustrate the major biological impact of frameshift acquisition. They demonstrate the importance of choosing the right strain or species for investigations of the function of a particular gene. However, it is not always possible to 
infer from the position of the frameshift whether the protein's activity will be affected. For instance, GlnA3, a glutamine synthetase generated from a frameshift-containing gene (Table 1), has been purified and shown to retain some activity [45]. It would be interesting to reframe these ORFs to test the impact of frameshift on protein function. On the other hand, it has been shown in silico that protein-coding sequences can be tolerant of frameshift translation events and thus that frameshit acquisition is an important reservoir for creating novel proteins [46]. Several of the truncated ORFs described here have also been detected in other studies, based on different analyses $[17,18,40,47,48]$. However, we present here a comprehensive comparative analysis of three related mycobacterial species and nine strains at the ICDS level.

We found no association between ICDS formation and mutation in the promoter region of the corresponding ORF. This suggests that promoter mutation and inactivation of gene expression are not the principal source of ICDS formation and hence of pseudogene accumulation in the M. tuberculosis complex. It may also suggest that ICDS formation in these species is a recent process. We favor the hypothesis that ORFs are first split into two or three parts, inactivating their function, and are then subject to secondary mutation (in both the ICDS and the untranslated region), leading to irreversible pseudogene fixation. Consistent with this hypothesis, we have observed additional mutations in the vicinity of the original frameshift in some strains.

We have shown that ICDS investigation can be used to infer the evolutionary relationships between strains and species. We provide here a list of more than 150 ICDSs that may be useful for characterizing TB strains and inferring phylogenetic relationships. The genome sequences of more than $10 \mathrm{~TB}$ strains will be released in the near future [26], and will, by no doubt, identify some new common and strain-specific ICDSs. Strain typing should clearly combine various markers, such as SNPs, MIRU, LSPs, RD, PE polymorphism [49] and ICDSs, in a matrix-based comparison from which the global phylogeny of TB isolates may be deduced. The polymorphism associated with these mutations is complementary to other methods $[17,34,36,37,50]$, hence can be used to explore genetic diversity within a given species. Interestingly, in strain 210, from the W-Beijing family, eight of the 19 ICDSs common to the five M. tuberculosis strains tested (H37Rv, CDC1551, Haarlem, F11, C) corresponded to full-length ORFs, illustrating its earlier divergence. Some of these genes may be involved in virulence, as they concern functions such as host cell invasion (ICDS0011 of H37Rv), lipid biosynthesis (ICDS0066 and ICDS0031 of H37Rv) and intermediary metabolism (ICDS0085 of H37Rv). To test whether this trait was a particularity of the 210 strain or applied more generally to the W-Beijing phylum, we sequenced these eight ORF that were full-length in this strain in 21 other clinical isolates of the W-Beijing (Table $3)$. In all cases, the ORF were corresponding to a fulllength ORF and not to an ICDS, demonstrating that these strains are genetically homogenous. The analysis performed using a strain of $M$. africanum showed that this species is characterized by an even fewer number of ICDSs common to M. tuberculosis H37Rv and CDC1551 than to the W-Beijing strains. More genome sequences of various strains and species are required for characterization of the genetic differences between the $\mathrm{W}$-Beijing strains and other species of the $M$. tuberculosis complex. The alkA gene has been shown to contain frameshift in both M. bovis and some M. tuberculosis isolates from Central African Republic [48]. The presence of SNPs in the adjacent region of the non-sense mutation has led the authors to propose a convergent evolution. Although, it probably depends from genes to genes, we instead favor the hypothesis that the non-sense mutation was acquired by the ancestor and spread to the progeny with acquisition of subsequent mutations in the adjacent region. Epidemiologists should bear in mind that a small percentage of ICDSs may correspond to sequencing errors $[4,11]$, generating artifactual genetic differences. Our analysis did not allow for the detection of mutations in which the frame of the coding sequence was conserved (synonymous mutation, in frame deletion), decreasing the total level of diversity observed. However, comparative ICDS analysis presents the major advantage of making it possible to associate the frameshift with a putative function and, possibly, with a particular phenotype. In conclusion, more attention should be paid to ICDS detection and comparison, particularly at the genomic scale.

\section{Conclusion}

We report here a comparative analysis of ICDSs in six isolates of $M$. tuberculosis, two of $M$. bovis and one of M. africanum. We show that these ICDSs can be classified as "common to all strains" or "strain-specific". Common ICDSs result from mutations acquired before the divergence of the species, whereas strain-specific ICDSs were acquired after this divergence. Comparative analyses of these ICDSs allow the definition of the molecular signature of a particular strain, phylogenetic lineage or species. We further show that ICDS formation is not correlated with the presence of a mutated promoter, and suggest that promoter extinction is not the main cause of pseudogene formation. The correlation between ICDSs, function and phenotypes could have important evolutionary implications and provides population geneticists with a list of targets, which could undergo selective pressure and thus alters relationships between the various lineages of $M$. tuberculosis strains and their host. 


\section{Methods \\ Databases}

The genome sequences of $M$. tuberculosis $\mathrm{H} 37 \mathrm{Rv}$ and CDC1551 and M. bovis AF2122/97 were taken from TIGR website [51]. The genome sequences of $M$. tuberculosis strains 210 or F11, C and Haarlem have been consulted on the TIGR or Broad Institute websites [52]. The genome sequence of $M$. bovis BCG $1173 \mathrm{P} 2$ has been taken from National Center for Biotechnology Information (NCBI) website (accession number, AM408590). The genome sequence of $M$. africanum GM041182 was consulted on line at the Sanger centre [53].

\section{Detection of common ICDS}

The genomic sequences of $M$. tuberculosis CDC1551, M. tuberculosis $\mathrm{H} 37 \mathrm{Rv}$, M. bovis AF2122/97 and M. bovis BCG 1173P2 have been scanned for couple of adjacent coding sequences that exhibit common homologs after translation. Such pair of coding sequences is considered as an ICDS if no paralogy relationship exists between the two coding sequences. The detailed description of ICDS detection is described in [3]. The ICDSs detected in each strain were then cross-compared by all-against-all blastn searches. For each ICDS, the best hits $\left(\mathrm{E}<10^{-65}\right)$ detected in the different strains were manually analysed to discriminate common and strain-specific ICDS.

\section{Sequencing analysis}

Chromosomal DNA of M. tuberculosis isolates from various lineages (Table 3) was used as a template for PCR amplification of the selected locus. The primers used to amplify and sequence were designed as previously described [3], using an optimized version of CADO4MI [54]. The nucleotide and deduced amino-acid sequences were analyzed with DNA Strider [55].

\section{Promoter analysis}

A region of 200 bp upstream the initiation codon was extracted for each of the 99 ICDSs specific to M. tuberculosis H37Rv, CDC1551 and M. bovis AF2122/97 (Additional files 2, 3 and 4). As a control group, 200 bp upstream the initiation codon was extracted for 99 genes (full-length) randomly selected from $M$. tuberculosis H37Rv. These 99 genes are full-length in M. tuberculosis H37Rv, CDC1551 and M. bovis AF2122/97. In each case (promoter to be tested and control group), the promoter regions of the 3 strains were aligned using ClustalW [56] and the sequence variation was recorded. The number of differences observed in the upstream region was statistically compared using the Chi2 test.

\section{Statistical analysis}

The statistical significance of the distribution of the frequency of sequence polymorphism observed in the upstream ICDS regions and upstream full-length regions, was tested using a Chi square test $\left(\mathrm{X}^{2}\right)$. The chi square test is used to determine relationship between two distributions. The calculated values were obtained: $X^{2}: 1,367, \mathrm{df}$ : 1, P value: 0.2423 , hence the difference between 2 groups are not statistically significant $(\alpha<0.05)$.

\section{Abbreviations}

ICDS, Interrupted CoDing Sequence. ORF, Open Reading Frame.

\section{Authors' contributions}

$\mathrm{CD}$ helped to carry out the bioinformatic studies, analysed the TB strains by sequencing and drafted the manuscript. EP carried out the bioinformatic studies and helped to draft the manuscript. DE analysed the TB strains by sequencing. EF helped to analyze the promoter regions. OP helped to draft the manuscript. PB participated in the analysis of the $\mathrm{W}$-Beijing strains and help to write the manuscript. OL participated in the design of the study, carried out the bioinformatic studies and drafted the manuscript. JMR conceived the study, participated in its design and coordination and in finalizing of the manuscript. All authors read and approved the final manuscript.

\section{Additional material}

\section{Additional file 1}

List of the ICDSs containing additional mutations in the $\mathrm{M}$. tuberculosis strains Haarlem, C and F11 with respect to H37Rv genome sequence.

Click here for file

[http://www.biomedcentral.com/content/supplementary/14712148-8-78-S1.doc]

\section{Additional file 2}

List of the ICDSs specific to M. bovis AF2122/97.

Click here for file

[http://www.biomedcentral.com/content/supplementary/14712148-8-78-S2.doc]

\section{Additional file 3}

List of the ICDSs specific to M. tuberculosis H37Rv.

Click here for file

[http://www.biomedcentral.com/content/supplementary/14712148-8-78-S3.doc]

\section{Additional file 4}

List of the ICDSs specific to M. tuberculosis CDC1551.

Click here for file

[http://www.biomedcentral.com/content/supplementary/14712148-8-78-S4.doc] 


\section{Additional file 5}

Nucleotide sequence differences of the upstream region (200 bp) of the Astrain specific ICDS B- the full-length genes (control group).

Click here for file

[http://www.biomedcentral.com/content/supplementary/14712148-8-78-S5.doc]

\section{Acknowledgements}

We thank R. Brosch and J. Belisle for the kind gift of chromosomal DNA. We thank F. Tekaïa for the help in statistical analysis. We thank S. Gagneux, P. M. Small, B. Gicquel, T. Dos Vultos and C. Sola for stimulating discussions and useful suggestions. We thank INSERM for funding this project under the Avenir program, through a grant to JMR, Chargé de Recherches at INSERM. This work was also funded by an RNG (Réseau National de Génopoles) grant to the Strasbourg Bioinformatics Platform infrastructures and EVIGENORET (LSHG-CT-2005-5I2036). CD is funded by a PhD grant from the Fondation pour la Recherche Médicale (FRM).

\section{References}

I. Cruveiller S, Le Saux J, Vallenet D, Lajus A, Bocs S, Medigue C: MICheck: a web tool for fast checking of syntactic annotations of bacterial genomes. Nucleic Acids Res 2005:W47I-479.

2. Liu Y, Harrison PM, Kunin V, Gerstein M: Comprehensive analysis of pseudogenes in prokaryotes: widespread gene decay and failure of putative horizontally transferred genes. Genome Biol 2004, 5(9):R64.

3. Perrodou E, Deshayes C, Muller J, Schaeffer C, Van Dorsselaer A, Ripp R, Poch O, Reyrat JM, Lecompte O: ICDS database: interrupted CoDing sequences in prokaryotic genomes. Nucleic Acids Res 2006:D338-343.

4. Deshayes C, Perrodou E, Gallien S, Euphrasie D, Schaeffer C, VanDorsselaer A, Poch O, Lecompte O, Reyrat JM: Interrupted coding sequences in Mycobacterium smegmatis : authentic mutations or sequencing errors? Genome Biol 2007, 8(2):R20.

5. Gomez-Valero L, Rocha EP, Latorre A, Silva FJ: Reconstructing the ancestor of Mycobacterium leprae: the dynamics of gene loss and genome reduction. Genome Res 2007, I 7(8): I I 78- I I 85.

6. Cole ST, Eiglmeier K, Parkhill J, James KD, Thomson NR, Wheeler PR, Honore N, Garnier T, Churcher C, Harris D, et al.: Massive gene decay in the leprosy bacillus. Nature 200I, 409(6823): $1007-1011$.

7. Stinear TP, Mve-Obiang A, Small PL, Frigui W, Pryor MJ, Brosch R, Jenkin GA, Johnson PD, Davies JK, Lee RE, et al.: Giant plasmidencoded polyketide synthases produce the macrolide toxin of Mycobacterium ulcerans. Proc Natl Acad Sci USA 2004 I 0 I(5): 1 345-1349.

8. Darby AC, Cho NH, Fuxelius HH, Westberg J, Andersson SG: Intracellular pathogens go extreme: genome evolution in the Rickettsiales. Trends Genet 2007, 23(10):51 I-520.

9. Guan X, Uberbacher EC: Alignments of DNA and protein sequences containing frameshift errors. Comput Appl Biosci 1996, I 2(I):31-40.

10. Hayashi K, Morooka N, Yamamoto Y, Fujita K, Isono K, Choi S, Ohtsubo E, Baba T, Wanner BL, Mori H, et al.: Highly accurate genome sequences of Escherichia coli K-I 2 strains MG 1655 and W3 I I O. Mol Syst Biol 2006, 2:2006 0007.

11. Medigue C, Rose M, Viari A, Danchin A: Detecting and analyzing DNA sequencing errors: toward a higher quality of the Bacillus subtilis genome sequence. Genome Res 1999, 9(II):III6-III.

12. $\mathrm{Xu} Y$, Mural RJ, Uberbacher EC: Correcting sequencing errors in DNA coding regions using a dynamic programming approach. Comput Appl Biosci 1995, I I (2): I I7-124.

13. Friedman CR, Quinn GC, Kreiswirth BN, Perlman DC, Salomon N, Schluger N, Lutfey M, Berger J, Poltoratskaia N, Riley LW: Widespread dissemination of a drug-susceptible strain of Mycobacterium tuberculosis. J Infect Dis 1997, 176(2):478-484.
14. Mathema B, Kurepina NE, Bifani PJ, Kreiswirth BN: Molecular epidemiology of tuberculosis: current insights. Clin Microbiol Rev 2006, 19(4):658-685.

15. Gutacker MM, Mathema B, Soini H, Shashkina E, Kreiswirth BN, Graviss EA, Musser JM: Single-nucleotide polymorphism-based population genetic analysis of Mycobacterium tuberculosis strains from 4 geographic sites. J Infect Dis 2006, 193(I): $121-128$.

16. Sreevatsan S, Pan X, Stockbauer KE, Connell ND, Kreiswirth BN, Whittam TS, Musser JM: Restricted structural gene polymorphism in the Mycobacterium tuberculosis complex indicates evolutionarily recent global dissemination. Proc Natl Acad Sci USA 1997, 94(18):9869-9874.

17. Filliol I, Motiwala AS, Cavatore M, Qi W, Hazbon MH, Bobadilla del Valle M, Fyfe J, Garcia-Garcia L, Rastogi N, Sola C, et al.: Global phylogeny of Mycobacterium tuberculosis based on single nucleotide polymorphism (SNP) analysis: insights into tuberculosis evolution, phylogenetic accuracy of other DNA fingerprinting systems, and recommendations for a minimal standard SNP set. J Bacteriol 2006, I 88(2):759-772.

18. Fleischmann RD, Alland D, Eisen JA, Carpenter L, White O, Peterson J, DeBoy R, Dodson R, Gwinn M, Haft D, et al:: Whole-genome comparison of Mycobacterium tuberculosis clinical and laboratory strains. J Bacteriol 2002, 184(19):5479-5490.

19. Manca C, Tsenova L, Barry CE 3rd, Bergtold A, Freeman S, Haslett PA, Musser JM, Freedman VH, Kaplan G: Mycobacterium tuberculosis CDC I55 I induces a more vigorous host response in vivo and in vitro, but is not more virulent than other clinical isolates. J Immunol 1999, I 62(I I):6740-6746.

20. Manca C, Tsenova L, Bergtold A, Freeman S, Tovey M, Musser JM, Barry CE 3rd, Freedman VH, Kaplan G: Virulence of a Mycobacterium tuberculosis clinical isolate in mice is determined by failure to induce ThI type immunity and is associated with induction of IFN-alpha/beta. Proc Natl Acad Sci USA 200I, 98(10):5752-5757

21. Reed MB, Domenech P, Manca C, Su H, Barczak AK, Kreiswirth BN, Kaplan G, Barry CE 3rd: A glycolipid of hypervirulent tuberculosis strains that inhibits the innate immune response. Nature 2004, 43 I (7004):84-87.

22. Camus JC, Pryor MJ, Medigue C, Cole ST: Re-annotation of the genome sequence of Mycobacterium tuberculosis H37Rv. Microbiology 2002, I 48(Pt I 0):2967-2973.

23. Cole ST, Brosch R, Parkhill J, Garnier T, Churcher C, Harris D, Gordon SV, Eiglmeier K, Gas S, Barry CE 3rd, et al.: Deciphering the biology of Mycobacterium tuberculosis from the complete genome sequence. Nature 1998, 393(6685):537-544.

24. Garnier T, Eiglmeier K, Camus JC, Medina N, Mansoor H, Pryor M, Duthoy S, Grondin S, Lacroix C, Monsempe C, et al.: The complete genome sequence of Mycobacterium bovis. Proc Natl Acad Sci USA 2003, I00(13):7877-7882.

25. Brosch R, Gordon SV, Marmiesse M, Brodin P, Buchrieser C, Eiglmeier K, Garnier T, Gutierrez C, Hewinson G, Kremer K, et al:: A new evolutionary scenario for the Mycobacterium tuberculosis complex. Proc Natl Acad Sci USA 2002, 99(6):3684-3689.

26. Bernal A, Ear U, Kyrpides N: Genomes OnLine Database (GOLD): a monitor of genome projects world-wide. Nucleic Acids Res 200I, 29(I): I 26-I27.

27. Brosch R, Gordon SV, Garnier T, Eiglmeier K, Frigui W, Valenti P, Dos Santos S, Duthoy S, Lacroix C, Garcia-Pelayo C, et al.: Genome plasticity of BCG and impact on vaccine efficacy. Proc Natl Acad Sci USA 2007, 104(13):5596-560I.

28. Oettinger $T$, Jorgensen $M$, Ladefoged $A$, Haslov $K$, Andersen $P$ : Development of the Mycobacterium bovis BCG vaccine: review of the historical and biochemical evidence for a genealogical tree. Tuber Lung Dis 1999, 79(4):243-250.

29. Brosch R, Gordon SV, Buchrieser C, Pym AS, Garnier T, Cole ST: Comparative genomics uncovers large tandem chromosomal duplications in Mycobacterium bovis BCG Pasteur. Yeast 2000, I7(2): III-123.

30. Pym AS, Brodin P, Brosch R, Huerre M, Cole ST: Loss of RDI contributed to the attenuation of the live tuberculosis vaccines Mycobacterium bovis BCG and Mycobacterium microti. Mol Microbiol 2002, 46(3):709-7I7.

31. Lopez B, Aguilar D, Orozco H, Burger M, Espitia C, Ritacco V, Barrera L, Kremer K, Hernandez-Pando R, Huygen K, et al.: A marked difference in pathogenesis and immune response induced by 
different Mycobacterium tuberculosis genotypes. Clin Exp Immunol 2003, I33(I):30-37.

32. Mira A, Pushker R: The silencing of pseudogenes. Mol Biol Evol 2005, 22(I I):2I35-2I38.

33. Bocs S, Danchin A, Medigue C: Re-annotation of genome microbial coding-sequences: finding new genes and inaccurately annotated genes. BMC Bioinformatics 2002, 3:5.

34. Goguet de la Salmoniere YO, Li HM, Torrea G, Bunschoten A, van Embden J, Gicquel B: Evaluation of spoligotyping in a study of the transmission of Mycobacterium tuberculosis. J Clin Microbiol 1997, 35(9):2210-2214.

35. Kamerbeek J, Schouls L, Kolk A, van Agterveld M, van Soolingen D, Kuijper S, Bunschoten A, Molhuizen H, Shaw R, Goyal M, et al: Simultaneous detection and strain differentiation of Mycobacterium tuberculosis for diagnosis and epidemiology. J Clin Microbiol 1997, 35(4):907-9|4.

36. Mazars E, Lesjean S, Banuls AL, Gilbert M, Vincent V, Gicquel B, Tibayrenc $M$, Locht $C$, Supply $P$ : High-resolution minisatellite-based typing as a portable approach to global analysis of Mycobacterium tuberculosis molecular epidemiology. Proc Natl Acad Sci USA 200I, 98(4): 190I-1906.

37. Kaser M, Rondini S, Naegeli M, Stinear T, Portaels F, Certa U, Pluschke G: Evolution of two distinct phylogenetic lineages of the emerging human pathogen Mycobacterium ulcerans. BMC Evol Biol 2007, 7(I): 177.

38. Constant P, Perez E, Malaga W, Laneelle MA, Saurel O, Daffe M, Guilhot C: Role of the pks I 5/I gene in the biosynthesis of phenolglycolipids in the Mycobacterium tuberculosis complex. Evidence that all strains synthesize glycosylated p-hydroxybenzoic methyl esters and that strains devoid of phenolglycolipids harbor a frameshift mutation in the pks I 5/I gene. Biol Chem 2002, 277(4I):38I48-38I58.

39. Gagneux S, DeRiemer K, Van T, Kato-Maeda M, de Jong BC, Narayanan S, Nicol M, Niemann S, Kremer K, Gutierrez MC, et al.: Variable host-pathogen compatibility in Mycobacterium tuberculosis. Proc Natl Acad Sci USA 2006, 1 03(8):2869-2873.

40. Tsolaki AG, Gagneux S, Pym AS, Goguet de la Salmoniere YO, Kreiswirth BN, Van Soolingen D, Small PM: Genomic deletions classify the Beijing/W strains as a distinct genetic lineage of Mycobacterium tuberculosis. I Clin Microbiol 2005, 43(7):3185-3191.

41. Reed MB, Gagneux S, Deriemer K, Small PM, Barry CE 3rd: The WBeijing lineage of Mycobacterium tuberculosis overproduces triglycerides and has the DosR dormancy regulon constitutively upregulated. J Bacteriol 2007, I 89(7):2583-2589.

42. Perez E, Constant P, Lemassu A, Laval F, Daffe M, Guilhot C: Characterization of three glycosyltransferases involved in the biosynthesis of the phenolic glycolipid antigens from the Mycobacterium tuberculosis complex. J Biol Chem 2004, 279(4I):42574-42583.

43. Boechat N, Lagier-Roger B, Petit S, Bordat Y, Rauzier J, Hance AJ, Gicquel B, Reyrat JM: Disruption of the gene homologous to mammalian NrampI in Mycobacterium tuberculosis does not affect virulence in mice. Infect Immun 2002, 70(8):4I 24-4I3I.

44. Domenech P, Reed MB, Barry CE 3rd: Contribution of the Mycobacterium tuberculosis MmpL protein family to virulence and drug resistance. Infect Immun 2005, 73(6):3492-350I.

45. Harth G, Maslesa-Galic S, Tullius MV, Horwitz MA: All four Mycobacterium tuberculosis gInA genes encode glutamine synthetase activities but only GlnAI is abundantly expressed and essential for bacterial homeostasis. Mol Microbiol 2005, 58(4): II57-| I 72.

46. Okamura K, Feuk L, Marques-Bonet T, Navarro A, Scherer SW: Frequent appearance of novel protein-coding sequences by frameshift translation. Genomics 2006, 88(6):690-697.

47. Marri PR, Bannantine JP, Golding GB: Comparative genomics of metabolic pathways in Mycobacterium species: gene duplication, gene decay and lateral gene transfer. FEMS Microbiol $\operatorname{Rev} 2006,30(6): 906-925$.

48. Nouvel LX, Dos Vultos T, Kassa-Kelembho E, Rauzier J, Gicquel B: A non-sense mutation in the putative anti-mutator gene adal alkA of Mycobacterium tuberculosis and $M$. bovis isolates suggests convergent evolution. BMC Microbiol 2007, 7:39.

49. Karboul A, Gey van Pittius NC, Namouchi A, Vincent V, Sola C, Rastogi N, Suffys P, Fabre M, Cataldi A, Huard RC, et al.: Insights into the evolutionary history of tubercle bacilli as disclosed by genetic rearrangements within a PE_PGRS duplicated gene pair. BMC Evol Biol 2006, 6: 107.

50. Read TD, Salzberg SL, Pop M, Shumway M, Umayam L, Jiang L, Holtzapple E, Busch JD, Smith KL, Schupp JM, et al.: Comparative genome sequencing for discovery of novel polymorphisms in Bacillus anthracis. Science 2002, 296(5575):2028-2033.

5I. J. Craig Venter Institute [http://www.tigr.org/]

52. The BROAD Institute [http://www.broad.mit.edu/tools/data/ seq.html]

53. Welcome trust Sanger Institute Mycobacterium africanum [http:/ /www.sanger.ac.uk/sequencing/Mycobacterium/africanum/].

54. Computer assisted Design of Oligonucleotide for Microarray [http://bips.u-strasbg.fr/CADO4MI/]

55. Marck C: 'DNA Strider': a 'C' program for the fast analysis of DNA and protein sequences on the Apple Macintosh family of computers. Nucleic Acids Res 1988, 16(5):1829-1836.

56. Thompson JD, Higgins DG, Gibson TJ: CLUSTAL W: improving the sensitivity of progressive multiple sequence alignment through sequence weighting, position-specific gap penalties and weight matrix choice. Nucleic Acids Res 1994, 22(22):4673-4680.
Publish with Bio Med Central and every scientist can read your work free of charge

"BioMed Central will be the most significant development for disseminating the results of biomedical research in our lifetime. "

Sir Paul Nurse, Cancer Research UK

Your research papers will be:

- available free of charge to the entire biomedical community

- peer reviewed and published immediately upon acceptance

- cited in PubMed and archived on PubMed Central

- yours - you keep the copyright
BioMedcentral 